\title{
Philosophiques
}

\section{Rationalité et sélection naturelle en économie}

\section{Maurice Lagueux}

Volume 25, numéro 2, automne 1998

Les modèles d'évolution en économie et en sciences sociales

URI : https://id.erudit.org/iderudit/027485ar

DOI : https://doi.org/10.7202/027485ar

Aller au sommaire du numéro

Éditeur(s)

Société de philosophie du Québec

ISSN

0316-2923 (imprimé)

1492-1391 (numérique)

Découvrir la revue

Citer cet article

Lagueux, M. (1998). Rationalité et sélection naturelle en économie. Philosophiques, 25(2), 163-180. https://doi.org/10.7202/027485ar

\section{Résumé de l'article}

Depuis la parution, en 1950, du célèbre article d'Armen Alchian, il est devenu assez fréquent d'invoquer la sélection naturelle pour appuyer certaines conclusions de l'économie néoclassique. Toute sélection n'étant toutefois pas de type " darwinien », il importe de bien distinguer les arguments qui invoquent la sélection naturelle au sens strict et les arguments crypto-téléologiques qui s'apparenteraientplutôt à un évolutionnisme de type lamarckien. A l'aide de quelques exemples fictifs, dont deux sont empruntés à un essai méthodologique de Milton Friedman de 1953, il est soutenu que les économistes doivent choisir entre des explications prétendument fondées sur la sélection naturelle et des explications fondées sur le principe de rationalité. Plus généralement, il est montré en conclusion, à l'aide de deux exemples supplémentaires, que les explications reposant sur des mécanismes impersonnels et celles reposant sur des activités intentionnelles ne peuvent être invoquées concurremment.
Ce document est protégé par la loi sur le droit d'auteur. L'utilisation des services d'Érudit (y compris la reproduction) est assujettie à sa politique d'utilisation que vous pouvez consulter en ligne.

https://apropos.erudit.org/fr/usagers/politique-dutilisation/ 


\title{
RATIONALIIÉ Et SÉlection MATURELLE EN ÉCONOMIE'
}

\author{
PAR \\ MAURICE LAGUEUX
}

RÉSUMÉ : Depuis la parution, en 1950, du célèbre article d'Armen Alchian, il est devenu assez fréquent d'invoquer la sélection naturelle pour appuyer certaines conclusions de l'économie néoclassique. Toute sélection n'étant toutefois pas de type "darwinien", il importe de bien distinguer les arguments qui invoquent la sélection naturelle au sens strict et les arguments cryptotéléologiques qui s'apparenteraient plutôt à un évolutionnisme de type lamarckien. À l'aide de quelques exemples fictifs, dont deux sont empruntés à un essai méthodologique de Milton Friedman de 1953, il est soutenu que les économistes doivent choisir entre des explications prétendument fondées sur la sélection naturelle et des explications fondées sur le principe de rationalité. Plus généralement, il est montré en conclusion, à l'aide de deux exemples supplémentaires, que les. explications reposant sur des mécanismes impersonnels et celles reposant sur des activités intentionnelles ne peuvent être invoquées concurremment.

ABSTRACT: Since the publication of Alchian's famous article of 1950, it has become relatively common to invoke natural selection as a justification of various conclusions in neoclassical economics. But because selection is not always of a "Darwinian" type, it is important to distinguish clearly between arguments which invoke natural selection in the strict sense of the word from cryptoteleological arguments which are rather more closely related to a Lamarkian evolutionary approach. With the help of fictional examples, two of which have been borrowed from Milton Friedman's 1953 essay on methodology, it is argued that economists must choose between explanations supposedly based on natural selection and ones that are based on the principle of rationality. More generally, and with the help of two further examples, it is concluded that explanations based on a anonymous mechanism and those based on intentional actisities can not be invoked concurrently.

1. Une première version de ce texte, rédigée en langue anglaise, est parue dans la revue Methodus (vol. $\mathrm{n}^{\circ} 1$, juin 1993, p. 93-100); elle avait préalablement été présentée aux sessions de l'International Network for Economic Method lors du congrès des Allied Social Science Associations tenu à Anaheim en janvier 1993. L'auteur remercie le CRSHC et le Fonds FCAR de leur assistance financière ainsi que D. Hammond, G. Lafleur, P. Mirowski, R. Nadeau, D. Ross, B. Toombs et les lecteurs anonymes de la revue Philosophiques de leurs très utiles commentaires. 
On a souvent souligné que l'analyse des phénomènes économiques repose, en dernier ressort, sur un postulat fondamental que l'on présente généralement comme le postulat de rationalité. C'est bien à une variante de ce postulat que se réfèrent les économistes quand ils semblent tenir pour acquis que les entrepreneurs prennent les décisions qui maximisent leur profit, compte tenu des contraintes auxquelles ils sont soumis. Or, tout au long de l'histoire de la pensée économique, ce postulat ainsi interprété a été l'une des cibles favorites des adversaires de la théorie économique. Comment prétendre que les entrepreneurs maximisent leur profit quand on a maintes fois observé que plusieurs d'entre eux, d'une part, connaissent fort mal divers paramètres affectant leur situation el, d'autre part, se laissent souvent guider par de tout autres considérations (prestige, maintien de bonnes relations, besoin de récupérer, etc.) quand ce n'est par leurs émotions ou par ce que Marx et Keynes appelaient plaisamment leurs "esprits animaux"? Il est difficile, en effet, de penser que les entrepreneurs ressemblent à ces machines à maximiser qu'invoquent volontiers les concepteurs de modèles économiques.

Diverses réponses ont été apportées aux objections de ce genre. Parmi ces réponses, celle qu'Armen Alchian a proposée en 1950 occupe une place à part et est connue des économistes sous le nom de survival argument ${ }^{2}$. Cet argument s'inspire explicitement de celui qui est à la base de la théorie darwinienne de la sélection naturelle. On peut résumer l'argument d'Alchian de la façon suivante. Quel que soit l'acharnement que les entrepreneurs pourraient mettre à maximiser leurs profits, ils doivent prendre leurs décisions dans un tel contexte d'incertitude qu'ils ont bien peu de chances de réaliser leurs objectifs. Toutefois, seuls ceux qui de facto auront pris des décisions qui ne sont pas trop éloignées de celles qui ont pour effet de maximiser ces profits se trouveront dans une situation financière susceptible, dans un marché compétitif, d'assurer leur survie économique. Ceux, au contraire, qui de facto auront fait les choix les plus éloignés de ceux qui leur auraient permis une telle maximisation risqueront d'être irrémédiablement voués à la faillite. Comme sur les marchés que les économistes analysent ne sauraient être présents que les entrepreneurs qui d'une façon ou d'une autre auront su éviter la faillite, ces entrepreneurs auront, dans l'ensemble, pris des décisions qui, de facto, auront été assez proches de celles qui maximisent les profits. Or', quand un économiste dit qu'un entrepreneur maximise ses profits, il ne se préoccupe pas de savoir ce que sont les intentions de cet entrepreneur, il lui suffit que celui-ci de facto maximise ces profits. Il est vrai que pour éviter la faillite, un entrepreneur n'a pas besoin de maximiser ses profits au sens strict puisqu'il lui suffit d'éviter d'encaisser des pertes trop importantes. Toutefois, un an

2. Armen A. Alchian, "Uncertainty, Evolution and Economic Theory ", Journal of Political Economy; vol. 58, 1950, p. 211-222. 
après la publication de l'article d'Alchian, Stephen Enke proposait une interprétation plus radicale du même argument ${ }^{3}$. Il rappelait que la théorie néoclassique établit que, dans un marché parfaitement compétitif, les entreprises doivent se contenter d'un profit nul qui, après comptabilisation de tous les coûts, correspond bel et bien au profit maximum et il en concluait que, à moins de maximiser effectivement leurs profits, ces entreprises encourent forcément des pertes et sont tôt ou tard vouées à la faillite. Que l'argument soit ainsi radicalisé ou non, Alchian et Enke estiment qu'il constitue une application légitime à l'économie de l'argumentation de Darwin.

Depuis la publication de ces textes, diverses objections ont été formulées à leur endroit ${ }^{4}$. Il ne sera toutefois pas question ici de reprendre le débat sur la validité de l'argument qu'ils proposent; il s'agira uniquement de discuter à son propos les deux questions suivantes : 1) cet argument est-il bien un argument similaire à celui de Darwin ou, si l'on préfère, met-il vraiment en ceuvre un mécanisme de sélection naturelle? 2) Un argument qui serait proprement darwinien serait-il compatible avec l'argument fondé sur le postulat de rationalité qu'invoquent habituellement les économistes? Ces deux questions sont plus étroitement interdépendantes qu'il ne semble car, comme on le verra, c'est précisément dans la mesure où le survival argument des économistes est interprété comme une application de la sélection naturelle - interprétation qui n'est toutefois pas la seule plausible - qu'il devient incompatible avec un argument fondé sur le principe de rationalité.

\section{Main invisible et sélection naturelle}

\section{Divers types de sélection}

Avant de voir si cet argument peut ou ne peut pas être considéré comme un argument darwinien, dégageons d'abord plus clairement le phénomène qu'il entend expliquer. Pour ce faire, il convient de considérer un univers économique où des entrepreneurs sont en concurrence, en ce sens que la part du marché contrôlée par l'un d'entre eux ne peut l'être par un autre, que les profits des uns se font le plus souvent aux dépens de ceux des autres et que, faute de parvenir à se tailler une part suffisante d'un marché forcément limité, chaque entreprise est, à plus ou moins long terme, menacée de faillite et donc d'extinction. Dans une telle situation, chaque entrepreneur

3. Stephen Enke, "On Maximizing Profits : A Distinetion between Chamberlin and Robinson ", American Economic Review, vol. 41, ${ }^{\circ} 4,1951$, p. 566-578.

4. Voir en particulier Edith T. Penrose, "Biological Analogies in the Theory of the Firm ^, American Economic Review, vol. 42, n' 5, 1952, p. 804-819 et Sidney G. Winter, "Economic "Natural selection" and the Theory of the Firm ", Fale Economic Essays, vol. 4, no 1, 1964, p. 242-272, pour une étude d'ensemble des relations entre l'économie et les théories de l'évolution en biologie, on pourra consulter Geoffrey M. Hodgson, Economics and Evolution : Bringing Back Life into Economics, Amn Arbour, University of Michigan Press, 1993. 
est amené, presque constamment, à prendre d'importantes décisions puisqu'il doit, selon les circonstances, investir ou désinvestir certains de ses actifs, augmenter ou réduire le niveau de sa production, congédier certains employés ou en embaucher de nouveaux, fixer le prix de ses produits, déterminer la quantité et la source de ses approvisionnements, etc. On voit mal comment on pourrait qualifier de particulièrement efficace le marché qui serait le lieu de toutes ces décisions indépendantes si d'aventure celles-ci devaient être prises au hasard. Admettons toutefois que les marchés qui sont observés de facto fonctionnent de façon passablement efficace, en ce sens que les marchandises les plus susceptibles de répondre aux besoins des acheteurs potentiels s'y trouvent généralement produites en quantités suffisantes et de manière assez rationnelle, compte tenu des ressources disponibles. Il convient donc d'expliquer cet état de choses, soit le fait que, même si aucune direction globale ne s'y exerce, un marché où chacun cherche son intérêt donne des résultats qui n'ont rien du chaos " économique » qui dominerait cet univers du moins est-on en droit de le penser - si les décisions y étaient prises au hasard.

L'explication de ce phénomène que fournit la théorie économique traditionnelle fait volontiers appel aux intentions des agents ou, si l'on préfère, aux fins poursuivies par ceux-ci. Elle postule que les entrepreneurs sont bien loin de s'en remettre au hasard et que, guidés par leur désir de réaliser des profits, ils s'efforcent, grâce à leur intelligence et à leur habileté (ou, si l'on préfère, à leur sens des affaires), de prendre les décisions qui leur paraissent les plus susceptibles de les mettre en position de maximiser ces profits. Cette théorie prétend ensuite montrer qu'un tel comportement de la part des entrepreneurs générera, pour l'ensemble de ce marché libre, un équilibre souvent qualifié d'optimal, mais ce n'est pas là l'aspect qui nous retiendra ici. Il nous suffira de noter que cette théorie postule de la part des entrepreneurs un comportement intentionnel et d'observer également qu'un tel comportement n'a de sens que dans un contexte foncièrement concurrentiel où il y a forcément des perdants et des gagnants. En ce sens, cette théorie suppose que les perdants seront exclus du jeu (de fait, ils sont toujours menacés de faillite) de telle sorte qu'il est légitime de dire que cette théorie suppose l'action d'une certaine sélection en vertu de laquelle ne sont retenus que les gagnants parmi tous les entrepreneurs potentiels qui auraient pu être attirés par ce jeu hautement concurrentiel.

Il paraît donc indiqué ici de se demander si un argument comme celui d'Alchian - interprété comme se référant à une sélection du type de celle décrite ci-dessus - a quelque chose à voir avec l'argument de Darwin basé sur la sélection naturelle. Pour répondre à une telle question, il faut d'abord se demander ce qui fait l'originalité de l'argument proposé par Darwin pour rendre compte de l'évolution 
des espèces. Il est bien connu que Darwin n'était pas le premier à soutenir que les espèces biologiques devaient être situées au sein d'un mouvement phylogénétique qui expliquait leur formation ; bien d'autres, comme Lamarck, l'avaient entrevu avant lui. Si, toutefois, la théorie de la sélection naturelle occupe la place que l'on sait dans l'histoire de l'évolution biologique, e'est qu'elle permettait de rendre compte de la formation d'espèces remarquablement bien adaptées sans faire appel, pour autant, à aucun type de finalité. Du point de vue scientifique, il était tout aussi inacceptable de faire appel à un Créateur dont les fins propres expliqueraient la formation des espèces que de prêter, de manière circulaire, à des espèces inférieures des fins qui permettraient de rendre compte de la genèse d'espèces dont, pour une large part, la supériorité tiendrait justement à leur aptitude à se donner de telles fins. Aussi, une théorie comme la sélection naturelle, qui faisait l'économie de toute forme de téléologie, constituait-elle pour la pensée scientifique un pas considérable, auquel est redevable la place exceptionnelle qui allait être celle de Darwin en histoire des sciences.

Pour faire ressortir l'originalité du mécanisme de sélection invoqué par Darwin et montrer qu'il faut se garder de le voir à l'cuvre dans n'importe quelle sorte de sélection, considérons la situation suivante. Supposons que divers candidats intéressés à quelques postes ouverts par une université prestigieuse se soumettent à un examen fort complexe imposé par le comité de sélection mis en place à cette université. Supposons aussi que la plupart de ces candidats obtiennent des résultats remarquables, mais que les rares postes ne sont finalement accordés qu'aux candidats (ou candidates) qui ont obtenu les meilleurs résultats. Imaginons maintenant qu'un observateur extérieur se présente alors et que, après s'être étonné de ce que les personnes retenues pour occuper ces postes soient d'un niveau intellectuel bien supérieur au niveau intellectuel moyen de la population, il demande que lui soit expliqué le fait qu'un résultat aussi heureux - et aussi statistiquement improbable s'il n'était dû qu'au hasard - ait pu être obtenu. Sans doute lui expliquera-t-on alors que ces personnes n'ont pas été choisies au hasard puisque, d'une part, celles qui se sont présentées à l'examen ont fait des efforts considérables pour optimiser leurs performances intellectuelles et que, d'autre part, le comité de sélection a intentionnellement choisi parmi ces personnes celles dont les performances étaient les plus remarquables. Dans ce processus, il y a done eu concurrence pour survivre et sélection des plus aptes, mais il serait évidemment absurde de soutenir que seul un recours à la sélection naturelle permet d'expliquer ce phénomène comme s'il eût fallu, n'eût été le génie de Darwin, le tenir pour inexplicable. On aura compris que la sélection à l'œuvre dans cet exemple n'a manifestement rien de darwinien puisqu'elle est de nature explicitement téléologique et cela, à un double niveau. D'une part, au niveau des individus, puisque seules se sont engagées dans le concours des personnes qui visaient à obtenir 
le meilleur résultat et prenaient les moyens nécessaires pour y arriver; d'autre part, au niveau du processus global, puisque les membres du comité de sélection qui visaient à ne retenir que les personnes les plus qualifiées ont fait les choix que requérait cet objectif. S'il fallait chercher parmi les théories de l'évolution biologique celle qui se rapproche le plus du processus considéré ici, il faudrait se tourner vers le créationnisme; en effet, c'est uniquement dans ce type d'explication qu'on a affaire à une sélection qui, au niveau du processus global, est effectuée intentionnellement, en l'occurrence par Dieu qui choisit parmi toutes les espèces possibles celles qui sont les mieux adaptées.

Mais revenons à l'explication de l'efficacité du marché que propose la théorie économique. Il s'agit là d'une explication qui est foncièrement différente de celle qui permet de rendre compte de l'heureux choix de notre comité de sélection parce que, au niveau global, la théorie économique n'invoque aucune sorte de finalité ou de décision intentionnelle. Toutefois, au niveau individuel, la finalité continue de jouer un rôle essentiel. Un entrepreneur décide, par exemple, d'investir une part de ses ressources dans la production de fromage plutôt que de les investir dans la production d'un autre produit ou encore de les conserver sous forme liquide dans l'attente de meilleures occasions parce qu'il estime que c'est là la décision qui risque de lui rapporter le plus. Mais cet entrepreneur n'est qu'un joueur parmi d'autres dans un jeu concurrentiel où le succès n'est garanti à personne. Tout ce que la théorie économique soutient, depuis Adam Smith, c'est que, nonobstant le fait qu'elles aient été prises sans que quiconque se soit préoccupé d'assurer le fonctionnement harmonieux du marché, les diverses décisions de ces entrepreneurs produisent justement ce résultat, un peu comme si une main invisible avait coordonné l'ensemble du processus. Or, ce que cette image de la main invisible veut mettre en relief, c'est que même si les fins poursuivies par les agents n'ont qu'une portée individuelle, un mécanisme d'un type particulier assure au nivean global un résultat tout aussi harmonieux que si Dieu - qui n'est évoqué ici que métaphoriquement - avait veillé intentionnellement à sa réalisation. C'est ce type d'explication qu'invoquent les économistes pour rendre compte du marché, mais un tel argument, on l'a vu, suppose un processus de sélection ${ }^{5}$. Si un résultat aussi harmonieux est obtenu, c'est que l'entrepreneur qui décide de produire du fromage à un moment où la demande ne justifie pas une croissance de la production de cet aliment risque d'encaisser des pertes susceptibles de le dissuader d'investir indéfiniment ses ressources là où elles ne sont pas requises. Or, il suffit que, dans ce jeu d'essais et d'erreurs, d'autres fassent beaucoup mieux que lui pour qu'il risque d'en payer

5. Pour une étude, devenue classique, des diverses caractéristiques des explications de type " main invisible ", on lira E. Ulmann-Margalit, "Invisible Hand Explanations „, Synthese, vol. 39, 1978, p. 263-291. 
le prix par la faillite, ce qui, en dernier ressort, contribuera au bon fonctionnement du mécanisme qu'évoque la métaphore de la main invisible. La théorie néoclassique, comme le rappelait l'argument de Enke, a même radicalisé cet état de choses, puisqu'elle suppose que les entrepreneurs s'efforcent de maximiser leurs profits, tout en concluant que ceux qui survivent ne peuvent, en principe, que produire à un niveau de profit égal à zéro. Bref, la théorie économique postule que le comportement des individus s'explique par leurs intentions (ou par leurs fins), mais elle conclut que, au niveau global, le résultat obtenu n'a rien d'intentionnel (ou de téléologique) et résulte plutôt d'une sélection qui s'opère dans le: contexte d'une concurrence qui ne pardonne pas. Mais ceci ne veut pas dire que le pouvoir explicatif de cette sélection soit de type darwinien pour autant.

Ici encore, s'il fallait trouver une théorie de l'évolution biologique qui se rapproche de l'analyse économique qui fait appel à la main invisible, il semble bien que la théorie retenue ne devrait pas être celle de Darwin. Mieux vaudrait, en effet, se toumer vers celle de Lamarck. Au niveau global, la théorie de Lamarck n'invoque aucune téléologie : ce sont des changements environnementaux aléatoires qui opèrent une sorte de sélection parmi les espèces dont les membres se sont efforcés d'améliorer leur adaptation à un environnement donné. Pas plus que chez Darwin, il n'est question ici d'une sélection intentionnelle comme celle qu'invoque le créationnisme. Par contre, au niveau des individus, ce sont les efforts, peut-être pas intentionnels, mais du moins orientés vers un but, qui expliquent l'adaptation progressive de ces individus à leur environnement ${ }^{6}$. Selon Lamarck, les ancêtres des girafes ont instinctivement cherché à étirer leur cou pour mieux atteindre les fruits disponibles sur des branches élevées et, cornme Lamarck croyait à tort que les caractères acquis étaient héréditaires, il pouvait expliquer ainsi la longueur du cou des giraf'es d'aujourd'hui. Même s'il entendait expliquer l'évolution par ce mécanisme d'adaptation et de transmission, Lamarck n'ignorait certes pas le fait que ce ne sont pas tous les descendants potentiels des girafes engagées dans ce processus qui étaient destinés à survivre ; aussi, puisqu'il estimait que l'étirement de leur cou était si important pour leur alimentation, devait-il bien se douter que les girafes qui parvenaient le plus efficacement à étirer le cou bénéficiaient d'un avantage appréciable sur les autres dans une jungle où tous les animaux sont constamment en lutte pour leur survie. On le voit, l'explication de l'évolution offerte par Lamarck est apparentée à l'explication du marché offerte par la théorie économique. Les entrepreneurs s'efforcent de s'adapter à leur environnement tout comme les girafes de Lamarck, mais comme ils

6. Pour les fins de la présente discussion, il importe peu que le comportement de ces individus soit intentionnel au sens strict ou qu'il soit purement instinctif; il suffit qu'il soit, en quelque façon, orienté vers un but. 
s'efforcent de le faire dans un contexte hautement compétitif, ils ne sont pas assurés du succès. Il y a parmi eux sélection des vainqueurs, mais cette sélection, qui tient largement aux aléas de l'environnement, n'est pas plus une sélection naturelle au sens darwinien que celle qui est implicitement à l'œuvre dans le mécanisme évolutif invoqué par Lamarck.

Qu'est-ce alors qui caractérise un mécanisme proprement darwinien? C'est, on l'a vu, l'absence de recours à la finalité, tant au point de vue individuel qu'au niveau global. Pour Darwin, les efforts déployés par les girafes pour étirer leur cou ne contribuent en rien à l'évolution de cette espèce. Si une quelconque girafe a pu épisodiquement jouer un rôle déterminant dans cette évolution, ce ne pouvait être, à ses yeux, qu'en vertu de circonstances accidentelles -en vertu d'accidents génétiques comme le préciseront les néodarwiniens - sans relation aucune avec quoi que ce soit qui puisse ressembler à un but ou à une intention. Bien sûr, pour que cet accident contribue à l'évolution de l'espèce, il fallait encore qu'il se révèle avantageux pour la survie et pour la procréation, mais l’important ici est que ce résultat pouvait être expliqué sans que soit invoqués les visées ou les efforts d'individus dont le résultat n'aurait pu, de toute façon, être transmis d'une génération à l'autre. En n'invoquant rien d'autre que des circonstances aléatoires, cette explication ne reposait en rien sur le succès plus ou moins grand obtenu par les individus dans leurs tentatives pour mieux s'adapter à leur environnement.

En économie, comme il s'agit de rendre compte du résultat d'une multitude d'actions humaines, le fait de se référer à des comportements orientés vers un but - et même à des comportements franchement intentionnels ou rationnels - ne pose évidemment pas les problèmes de principe auxquels on se heurtait en biologie. Aussi n'est-il pas surprenant que, à une seule exception près, on ne rencontre pas, du moins dans la littérature influente, de tentatives de rendre compte des phénomènes économiques qui remettent radicalement en question le rôle dévolu aux comportements présumés rationnels. Alchian lui-même, tout convaincu qu'il ait été du caractère darwinien de son argument, ne mettait pas vraiment en cause le caractère intentionnel du comportement des entrepreneurs. L'unique exception à laquelle je viens de faire allusion est le texte assez étonnant que Gary Becker a consacré à la mise au point d'un modèle économique fondé sur des comportements totalement irrationnels?. Dans ce texte, Becker soutient qu'il est possible de dériver les principales conclusions de la microéconomie sans faire appel au postulat de rationalité. Pour le montrer, il construit un modèle où n'interviennent que des individus

7. Gary S. Becker, " Irrational Behavior and Economic Theory ", Journal of Political Economy, vol. 70, février 1962, p. 1-13. 
irrationnels en ceci que les comportements qui leur sont imputés ne dépendent en aucune façon des caractéristiques de la situation dans laquelle ils s'inscrivent, puisque ou bien ils sont strictement déterminés au hasard, ou bien ils consistent à répéter mécaniquement et aveuglément un même comportement initial. La plupart des commentaires portant sur cette entreprise audacieuse ont mis fortement en doute sa capacité à dériver des conclusions économiques vraiment significatives ${ }^{8}$, mais ce qu'il importe de voir ici, c'est que, s’il eût été satisfaisant, le modèle de Becker aurait constitué une explication proprement darwinienne des phénomènes économiques. En effet, cette explication aurait, tout comme celle proposée par Darwin, permis de rendre compte d'un phénomène remarquablement organisé (soit, respectivement, un marché libre qui fonctionne de manière relativement efficace ou une espèce étonnamment bien adaptée à son environnement) sans faire appel à aucune forme de finalité, pas plus au niveau des individus qu'au niveau global.

\section{Les joueurs de billard et les feuilles calculatrices}

Pour mieux cerner la différence entre ces divers types d'explication, il peut être utile de considérer deux des pittoresques exemples que Milton Friedman a proposés dans sa célèbre intervention méthodologique ${ }^{9}$ de 1953 . L'un d'entre eux, explicitement associé par Friedman à l'argument d'Alchian, concerne la densité des feuilles autour d'un arbre; l'autre, qui n'est pas mis en rapport avec cet argument, met en scène des experts au jeu de billard.

Examinons d'abord ce dernier argument. Supposons avec Friedman qu'il existe une formule mathématique complexe - par exemple, une formule de nature trigonométrique - qui permettrait de déterminer la trajectoire exacte d'une boule de billard devant en frapper une autre de façon optimale et supposons que les experts à ce jeu sont totalement incapables de maîtriser cette formule mathématique dont ils ne soupçonnent même pas l'existence. Cos joueurs n'en sont évidemment pas réduits pour autant à frapper la boule au hasard. Tout comme les entrepreneurs les plus habiles, ils utilisent au mieux leurs connaissances et leur savoir-faire pour atteindre leur but. Naturellement, certains joueurs réussissent mieux que d'autres, mais seuls ceux qui, d'une manière ou d'une autre, parviennent à atteindre un résultat qui pour l'essentiel coïncide avec celui que prédisait la formule mathématique méritent d'être qualifiés

8. J'ai eu l'occasion de discuter ce texte et les débats qu'il a suscités dans l'article intitulé "Kirzner versus Becker : Rationality and Mecharrisms in Economics " paru dans Robert F. Hébert (dir.), Perspectives on the History of Economic Thought, vol. IX. Themes on Economic Discourse, Method, Money and Trade. Aldershot, England, 1993, p. 37-50.

9. Milton Friedman, Essays in Positive Economics, Chicago, The University of Chicago Press, 1953. 
de joueurs experts. Friedman avait tort, à mon sens, de penser que cet exemple pouvait permettre de conclure que le réalisme des postulats importe peu ${ }^{10}$, mais il avait parfaitement raison, me semble-t-il, de penser que cet exemple illustre fort bien la façon dont la théorie économique néoclassique prétend rendre compte du fonctionnement du marché. La théorie économique néoclassique, tout comme la théorie mathématique du jeu de billard, vise à analyser ce qui se passe quand des agents tentent intentionnellement d'atteindre leurs buts respectifs dans un jeu hautement concurrentiel. La réussite ne peut évidernment être garantie à tous les agents, mais la théorie suggère que ceux d'entre eux dont les efforts seront d'une manière ou d'une autre couronnés de succès auront agi de facto d'une façon dont la logique est analysée par la théorie. Dans un cas comme dans l'autre, l'argument suppose que les agents qui auront posé des gestes trop différents de ceux qu'exige la théorie seront exclus de ce jeu concurrentiel. C'est bien là ce que laisse entendre l'économiste néoclassique quand il dit qu'un entrepreneur ne peut vendre à tel ou tel prix, car alors il produirait à perte ; en effet, cet économiste ne prétend pas que l'entrepreneur n'a pas la liberté de vendre à ce prix, il soutient seulement que s'il continue de le faire pendant un certain temps, il sera tôt ou tard mené à la faillite et exclu du marché. Dans les jeux concurrentiels comme l'économie de marché ou le billard, il y a forcément des gagnants et des perdants ou, si l'on préfère, il y a sélection. Mais comme cette sélection résulte simplement du fait quiil y a des gagnants dans le combat pour la vie dans lequel un certain nombre d'individus s'engagent avec l'intention ferme de gagner, cette sélection a peu à voir avec le mécanisme explicatif invoqué par Darwin.

Pour illustrer, sans trop s'éloigner de cet exemple, ce que pourrait être un mécanisme proprement darwinien, le jeu de billard "standard" évoqué jusqu'ici devrait être remplacé par ce que j'appellerai un jeu de billard a labyrinthin ». Dans ce jeu, qui paraîtra sans doute un peu bizarre et assez peu attrayant, on suppose que la table est transformée en un labyrinthe extrêmement complexe, au point où les joueurs les plus experts se disent parfaitement indifférents à pousser les boules dans une direction ou dans une autre, puisque celles-ci empruntent, de toute façon, des trajectoires qu'il leur est totalement impossible de prévoir. Supposons néanmoins que ce jeu est concurrentiel au sens où les joueurs qui ne parviennent pas à diriger suffisamment de boules dans les sacs qui servent de cibles sont éliminés. Dans ces conditions, il suffit de supposer que chaque joueur adopte pour stratégie de toujours frapper la boule de la même façon, une fois qu'il a déterminé, purement au hasard, son premier coup, pour qu'il soit possible en

10. Voir à ce sujet Maurice Lagueux, "Friedman's "Instrumentalism " and Constructive Empiricism in Economics ", Theory and Decision, vol. 37, 1994, p. 147-174. 
principe d'expliquer à l'aide d'une forme (minimale) de sélection naturelle que, après un certain temps, ceux qui a survivent »à ce jeu frappent la balle de façon efficace et de façon somme toute assez conforme à ce qu'exigeait la formule mathématique qu'aurait mise au point l'inventeur du jeu. Par hypothèse, c'est le conservatisme viscéral des joueurs favorisés par la chance qui entraînerait ici la transmission, d'un coup à un autre et d'une joute à une autre, de façons de faire qui, par hasard et par accident, se seraient révélées relativement adaptées. Si la structure du jeu devait se modifier très lentement et exiger ainsi des coups quelque peu différents, il suffirait de supposer que, de manière accidentelle et exceptionnelle, un coup soit, de temps à autre, déterminé au hasard avant d'être repris durant une longue séquence de coups successifs pour que certains des joueurs survivants dans un interminable toumoi voient leurs coups peu à peu réadaptés aux exigences du jeu. Bref, c'est une sorte de sélection naturelle qui ferait surgir de la sorte un groupe bien peu glorieux de " joueurs experts ».

La situation serait toutefois fort différente si quelques joueurs particulièrement astucieux imaginaient une façon de déjouer le concepteur du jeu et parvenaient, grâce à de savantes combines, à déterminer la bonne façon de jouer. Dans le cas où les joueurs sen remettraient au hasard, la sélection naturelle permettrait d'expliquer l'efficacité relative de joueurs qui n'étaient même pas en mesure de viser quoi que ce soit. Dans le cas où certains joueurs trouveraient une façon de déjouer le concepteur du jeu et de se montrer plus efficaces que ceux qui jouent au hasard (à défaut de quoi ils n'auraient rien déjoué du tout), leur efficacité (d'un autre ordre) ne s'expliquerait plus par la sélection naturelle ; elle s'expliquerait par le fait qu'ils auraient intentionnellement trouvé des moyens d'arriver $\grave{a}$ leur fin. Loin de voir leur remarquable succès expliqué par la sélection naturelle, les gagnants de ce jeu ne pourraient être considérés tels que dans la mesure où ils auraient su dépasser franchement les résultats que la sélection naturelle aurait permis d'espérer.

Considérons maintenant l'autre exemple proposé par Friedman, soit celui concernant la densité des feuilles autour d'un arbre, exemple que son auteur met lui-même en rapport avec unc explication fondée sur la sélection naturelle. Dans cet exemple, le fait (tenu hypothétiquement pour vrai) que les feuilles sont distribuées autour de cet arbre exactement " comme si chaque feuille avait délibérément cherché à maximiser la quantité de lumière qu'elle reçoit $^{11}$ »est expliqué sans faire appel à un processus intentionnel de maximisation qui, en la circonstance, paraîtrait plutôt incongru. Voyons comment Friedman présente la chose : " Nous sommes enclin à "expliquer" sa validité par le fait que la lumière du soleil contribue

11. Friedman, Essays in Positive Economics, p. 19 (traduction libre). 
à la croissance des feuilles et que, par là même, le feuillage s'en trouve plus dense ou, si l'on préfère, plus de feuilles parviennent à survivre là où il y a plus de soleil, de telle sorte que le résultat atteint par une adaptation purement passive aux circonstances extérieures est le même que le résultat qui prévaudrait en vertu d'un ajustement délibéré à celles-ci ${ }^{12}$. Avons-nous affaire ici à une explication proprement " darwinienne " ou plutôt à une explication qui se rapproche encore de celle proposée par Lamarck ? La réponse à cette question ne va pas de soi. La notion d' $\propto$ adaptation aux circonstances externes " évoque au premier abord l'approche de Lamarck, mais, chez ce dernier, l'adaptation n'est pas " purement passive ». Chez Darwin, par ailleurs, l'adaptation ne se réalise " passivement" que par l'intervention d'un mécanisme qui n'est pas clairement évoqué ici. Un argument proprement darwinien consisterait, par exemple, à rendre compte de l'existence d'un type d'arbre dont les feuilles seraient distribuées de manière à capter le maximum de lumière solaire. Pour expliquer ce phénomène, il faudrait toutefois faire appel, non pas simplement à une adaptation passive, mais à certaines variations accidentelles dont le résultat, par pur hasard, se serait révélé favorable et se serait transmis fidèlement, avec un gage accru de survie, de génération en génération. Cette explication, on le voit, s'apparente à celle - basée sur des modifications aléatoires de stratégies qui seraient transmises ensuite avec une fidélité aveugle que j'ai proposée plus haut pour rendre compte (dans certaines conditions hypothétiques fort restrictives) du succès de certains joueurs au billard labyrinthin ou de l'efficacité d'un marché animé par les agents irrationnels imaginés par Becker.

Bref, ce qui fait l'originalité d'un argument proprement darwinien n'est pas le simple fait que les feuilles tendent, plus ou moins passivement, à être plus nombreuses du côté ensoleillé d'un arbre que de son côté ombragé. Quiconque entend expliquer le fait irrécusable que le blé se retrouve en bien plus grande quantité dans les terres riches en éléments nutritifs que dans les terres rocailleuses, n'a pas besoin d'attendre le génie de Darwin pour invoquer le fait comme le faisait déjà, implicitement, une parabole de l'Évangile fort connue - que le blé ne peut survivre dans les terres du deuxième type précisément à cause de l'absence d'éléments nutritifs. De même, on pouvait comprendre bien avant Darwin - un biologiste lamarckien, en tout cas, n'aurait certes pas eu de mal à l'expliquer qu'il y ait plus de feuilles sur le côté ensoleillé d'un arbre que sur son côté ombragé. Dans un tel cas, les feuilles se retrouvent du côté qui leur est favorable comme si elles avaient choisi intentionnellement cette position, mais il n'est pas besoin de faire appel à la logique de la sélection naturelle de Darwin pour montrer que ce phénomène n'implique pas pour autant que les feuilles soient en mesure de

12. Ibid., p. 20 (traduction libre). 
s'adonner à de savants calculs; il suffit d'admettre que ces feuilles ne parviennent aisément à se développer en abondance que là où elles peuvent bénéficier de la présence du soleil.

On peut, à l'aide du tableau qui suit, caractériser les divers types d'arguments considérés ici. La catégorisation qui y est proposée ne vise nullement à établir des distinctions étanches entre des théories biologiques et pas davantage à proposer des parallèles étroits entre ces dernières et des théories économiques; elle invite plutôt à bien distinguer divers types d'explication que peuvent invoquer les économistes quand ils recourent à des modes d'argumentation que les théories de l'évolution biologique ont grandement contribué à populariser.

\begin{tabular}{|c|c|c|c|c|}
\hline \multirow[t]{2}{*}{$\begin{array}{l}\text { Théorie } \\
\text { économique }\end{array}$} & \multirow[t]{2}{*}{$\begin{array}{l}\text { Exemples } \\
\text { fictifs }\end{array}$} & \multirow[t]{2}{*}{$\begin{array}{l}\text { Théories sur } \\
\text { l'origine des } \\
\text { espèces } \\
\text { biologiques }\end{array}$} & \multicolumn{2}{|c|}{$\begin{array}{l}\text { Comportement intentionnel } \\
\text { (ou orienté vers un but) }\end{array}$} \\
\hline & & & $\begin{array}{c}\text { niveau des } \\
\text { individus }\end{array}$ & niveau global \\
\hline & $\begin{array}{l}\text { Comité de } \\
\text { sélection } \\
\text { universitaire }\end{array}$ & & Oui & Oui \\
\hline & & Créationnisme & ...... & Oui \\
\hline $\begin{array}{l}\text { Théorie } \\
\text { néoclassique de } \\
\text { la firme dans } \\
\text { un marché } \\
\text { concurrentiel } \\
\text { (main invisible) }\end{array}$ & $\begin{array}{l}\text { Experts au } \\
\text { billard selon } \\
\text { Friedman } \\
\text { Feuilles } \\
\text { croissant au } \\
\text { soleil selon } \\
\text { Friedman } \\
\end{array}$ & Lamarckisme & Oui & Non \\
\hline $\begin{array}{l}\text { Modèle } \\
\text { économique } \\
\text { avec agents } \\
\text { irrationnels au } \\
\text { sens de Becker }\end{array}$ & $\begin{array}{l}\text { Billard } \\
\text { labyrintin } \\
\text { Type d'arbre } \\
\text { aux feuilles } \\
\text { optimalement } \\
\text { orientées (selon } \\
\text { Friedman?) }\end{array}$ & Darwinisme & Non & Non \\
\hline
\end{tabular}

Si l'on veut se référer à l'exemple friedmanien des feuilles d'arbre pour illustrer le mécanisme à l'œuvre dans un marché économique, il faut donc opter entre deux façons d'interpréter l'adaptation qui en constitue l'élément clé. Ou bien on a affaire à un processus actif (les feuilles croissent là où elles le peuvent) et alors on est plus près du 
lamarckisme que du darwinisme. Ou bien on a affaire à un processus strictement passif (l'arbre est accidentellement doté de tel caractère qui se révèle avantageux) et c'est en cela que l'explication apportée est proprement darwinienne ${ }^{13}$. Si l'on revient à l'économie, la première interprétation correspondrait à une situation où les entrepreneurs cherchent activement à s'adapter à leur environnement en vue d'accroître leurs profits, de telle sorte que ceux qui ont le plus de succès à ce jeu se retrouvent dans une position relativement avantageuse que la théorie néoclassique cherche à déterminer. Dans le second cas, l'on admet que les entrepreneurs ne peuvent guère, vu le contexte r'incertitude dans lequel ils évoluent, contribuer de façon significative à améliorer leur situation, mais grâce à un argument d'allure héroïque, du type de celui qu'invoquait Becker, on n'en maintient pas moins que les entrepreneurs qui auront la chance de survivre dans un marché donné se retrouveront dans la situation globalement décrite par la théorie économique.

\section{Deux modes d'explication incompatibles}

Reste à savoir si ces deux interprétations sont compatibles entre elles. Â première vue, on pourrait penser qu'elles le sont. Une société économique est, en effet, une réalité fort complexe où l'on rencontre des entrepreneurs fort habiles et fort soucieux de tout mettre en cuvre pour accroître leurs profits et d'autres plus erratiques et sonvent moins motivés. Pourquoi le succès des premiers ne s'expliquerait-il pas par un argument reposant sur la rationalité de leurs actions, alors même que la survie des seconds pourrait s'expliquer par une application de la sélection naturelle à l'économie ? Le problème que pose cette sorte d'éclectisme tient au fait que, en principe, tous ces entrepreneurs sont en concurrence sur un même marché. S'il se trouve que les entrepreneurs les plus motivés et les phus astucieux tendent, en vertu même de ces qualités, à l'emporter sur ceux qui le sont moins, lesquels risquent, de ce fait, d'êlre carrément exchus du marché, alors la sélection qui s'effectue entre eux est bien celle que suppose la théorie économique du marché concurrentiel et ne repose en rien sur un mécanisme de sélection naturelle. Si, par contre, il se trouve que le contexte dans lequel évoluent les entrepreneurs est tellement incertain et aléatoire que ceux-ci sont littéralement incapables, quelles que soient leur habileté et leur motivation, de contribuer vraiment à améliorer leur situation, alors il n'y a aucun sens à invoquer une forme quelconque

13. La plupart des spécialistes reconnaissent que l'adaptation résultant de la sélection naturelle n'est pas forcément optimale et il n'est pas question ici de dénier ce point, d'autant plus que le présent argument suppose justement que lés phénomènes humains et sociaux donnent lieu à certaines formes d'adaptation que la sélection naturelle ne saurait expliquer. 
d'intentionnalité pour expliquer ce dont la sélection naturelle suffit à rendre compte.

La question essentielle est celle de savoir si ce qu'il s'agit d'expliquer dépasse ou non ce dont un mécanisme non intentionnel permet de rendre compte. Par exemple, dans le jeu de billard labyrinthin imaginé plus haut, la question est de savoir si certains joueurs peuvent ou non, à force d'habileté ou de ruse, trouver une façon d'arriver plus rapidement aux résultats visés que ceux qui se contentent de se laisser guider par le hasard. Si l'on répond par l'affirmative à cette question, alors on a simplement affaire à un jeu d'adresse où toutefois les performances requises sont plus difficiles à réaliser que celles normalement associées au jeu de billard. Dans ce nouveau jeu, des experts finissent par s'imposer comme dans tous les jeux. Tout au plus, ces experts doivent-ils partager parfois les meilleures places avec d'autres qui sont moins habiles mais qui, en ces occasions, ont eu passablement de chance. On ne voit donc pas en quoi les remarquables performances observées dépendraient de la sélection naturelle. Si, par contre, on doit répondre à la question par la négative en estimant qu'on ne peut dans ce jeu faire mieux que le hasard, alors il n'y a pas de raison de s'attendre à observer des performances particulièrement efficaces, à moins que la sélection naturelle n'ait pu faire son cuvre. Il n'est, en effet, pas possible de reconnaître un rôle explicatif aux décisions intentionnelles des joueurs puisque, par hypothèse, celles-ci ne comptent pour rien, au point où les joueurs sont totalement indifférents à viser dans une direction plutôt que dans une autre.

Bref, ces deux sortes d'explication (individus rationnels entrant en concurrence et sélection naturelle) sont vouées respectivement à expliquer des phénomènes différents ou, mieux, des situations différentes et c'est en ce sens qu'en tant qu'explication d'un phénomène elles sont incompatibles entre elles. On sera amené à invoquer l'une ou l'autre selon que le phénomène à expliquer est tel ou tel. En économie, ou bien il s'agit d'expliquer l'efficacité étonnante qui, hypothétiquement, résulterait des actions totalement irrationnelles d'entrepreneurs dont les calculs seraient parfaitement vains dans le contexte d'incertitude où ils seraient plongés, et alor's on doit invoquer un mécanisme de sélection naturelle comme celui qui est à l'œuvre dans l'interprétation que j'ai proposée du modèle de Becker. Ou bien il s'agit d'expliquer qu'un marché où évoluent librement des entrepreneurs rationnels produit des résultats jugés supérieurs (par exemple, en termes de degré de précision ou de rapidité d'adaptation) à ce que la sélection naturelle permettrait d'expliquer, et alors c'est vers la rationalité des décideurs qu'il faut se tourner pour expliquer le phénomène. Bref, si les entrepreneurs ne peuvent faire mieux que ce qui peut être obtenu en agissant au hasard, alors il n'y a rien à expliquer à l'aide d'une autre cause que la sélection naturelle et s'ils peuvent faire mieux, il n'y a pas lieu de 
chercher à expliquer par la sélection naturelle ce que celle-ci, par hypothèse, ne saurait expliquer. Il n'y a donc pas de sens à mêler ces deux types d'explication. Plus précisément, les invoquer concurremment à propos du même phénomène équivaudrait à subordonner l'une à l'autre, à désactiver l'une au profit de l'autre ou, mieux, à réinterpréter la première dans les termes de la seconde. Imaginer que les entrepreneurs prennent rationnellement les moyens d'arriver à un résultat qui aurait été obtenu de toute façon par sélection naturelle (en supposant qu'une telle chose soit pensable), ce ne serait pas apporter deux explications complémentaires du même phénomène, ce serait enlever toute portée à la première explication et la réinterpréter dans les termes de la seconde : en se donnant l'impression de faire des choix réfléchis, les entrepreneurs n’auraient rien fait d'autre, objectivement, que de choisir au hasard, puisque tous les choix possibles auraient été rigoureusement équivalents quant au résultat espéré.

On pourrait être enclin à objecter ici que les deux explications peuvent néanmoins être complémentaires dans la mesure où la sélection naturelle expliquerait le fait que le marché fonctionne efficacement et parvient ainsi à satisfaire les besoins sociaux, alors que le comportement rationnel de certains entrepreneurs expliquerait que ce soit eux, plutôt que leurs collègues moins rationnels, qui parviennent à réaliser les profits supposés par ce bon fonctionnement. Ce serait cependant confondre la sélection naturelle et cette sorte de sélection qui, comme on l'a vu, a forcément cours même quand la concurrence oppose des entrepreneurs rationnels, comme le veut la théorie économique depuis Adam Smith. Ou bien les entrepreneurs cherchent rationnellement à accroître leurs profits et les plus aptes d'entre eux, qui y parviennent le plus efficacement, sont alors sélectionnés par le jeu de la concurrence - par la main invisible si l'on préfère -, mais cette sélection qui résulte d'actes rationnels n'a rien d'une sélection de type darwinien; ou bien c'est une véritable sélection naturelle qui agit sur des individus irrationnels au sens où les définit Becker, mais alors le succès des individus sélectionnés n'est nullement dû̀ ù un comportement rationnel puisque, par hypothèse, leur comportement n'a rien de rationnel.

On peut, en terminant, illustrer plus clairement peut-être l'incongruité qu'il y aurait à additionner deux explications de natures aussi différentes à l'aide d'un exemple plus simple en ceci que le phénomène à expliquer n'y comporte qu'un seul aspect par opposition aux deux aspects (efficacité globale du marché et succès individuel des entrepreneurs) de l'exemple précédent. Si l'on suppose que des archers parviennent avec un succès étonnant à atteindre une cible fort éloignée, on expliquera normalement ce succès en invoquant leur détermination, leur habileté et les moyens rationnels qu'ils ont pris pour réussir de telles performances. Si 
toutefois on découvre, plus tard, qu'un aimant extrêmement puissant était caché derrière le point central de la cible et qu'il attirait aisément toutes les flèches volant dans les environs, alors le phénomène est expliqué par le champ magnétique créé par la présence de cet aimant et il ne reste plus rien à expliquer par la détermination et la rationalité des archers. Puisque, à l'intérieur de certaines limites, la direction dans laquelle les flèches sont lancées n'a absolument aucun impact sur le résultat observé, on voit mal ce que l'habileté des archers pourrait contribuer à expliquer. II n'est plus question ici d'expliquer la remarquable performance d'archers experts puisqu'il n'y a rien de tel à expliquer de la part de ces archers qui n'ont rien réussi de plus que le plus malhabile des individus qui décocherait une flèche dans ces conditions. Sans doute, si le pouvoir magnétique de l'aimant venait à faire défaut, les flèches des experts continueraient à se diriger au centre de la cible et il faudrait alors expliquer la chose en se basant sur des phénomènes intentionnels, mais e'est qu'il y aurait désormais un nouveau phénomène à expliquer, soit le fait que les flèches des experts atteignent systématiquement un point que les flèches lancées nonchalamment n'atteignent désormais à peu près jamais plus.

En somme, quand un mécanisme général, pen importe qu'il sagisse du magnétisme ou de la sélection naturelle, peut expliquer un état de chose, il n'y a aucun sens à invoquer des explications intentionnelles pour en rendre compte. Si un individu décide de se suicider et se laisse tomber du sommet d'un édifice, sans doute faut-il invoquer une explication intentionnelle pour expliquer sa décision et son geste fatal. Mais si tout au cours de sa chute, il fait tout ce qui est en son pouvoir pour arriver jusqu'au sol de manière à se fracasser la tête et ainsi atteindre sa fin, il n'y a pas lieu de chercher là une explication du fait, rapidement vérifié, qu'il y arrive effectivement, puisque la gravitation universelle y suffit amplement. Ce n'est qu'au moment où ces mécanismes généraux sont impuissants à expliquer un phénomène qüil y a lieu de recourir à une explication intentionnelle (détermination et rationalité des archers ou des entrepreneurs, désespoir et détermination du suicidé, etc.) pour en rendre compte. Or, comme il se trouve que ce sont de tels phénomènes que les sciences sociales - et l'économie en particulier - sont appelées à expliquer, on ne peut, dans ces sciences, invoquer l'efficace de ces mécanismes généraux à moins de dissoudre littéralement le problème qu'il s'agit d'expliquer. Bref, si la sélection naturelle et l'action rationnelle (maximisatrice ou pas) sont des explications incompatibles, c'est qu'elles ne rendent pas compte de phénomènes du même ordre. S'il y a vraiment lieu d'invoquer la sélection naturelle ou la gravitation universelle pour expliquer un phénomène, c'est que les conditions qui pourraient donner lieu à une explication basée sur l'action rationnelle ne sont pas des composantes significatives de ce phénomène. Et si de telles conditions sont absentes, c'est qu'on n'a tout simplement pas affaire à un phénomène 
proprement social qu'il conviendrait d'expliquer à l'aide d'une analyse des conséquences complexes de multiples décisions humaines, comme celle que la science économique a toujours prétendu nous apporter.

Département de philosophie

Université de Montréal 